\title{
O DESENVOLVIMENTO PSICOSSOCIAL E EDUCACIONAL DE INDIVÍDUOS COM ANOMALIAS FACIAIS
}

\author{
PSYCHOSOCIAL AND EDUCATIONAL DEVELOPMENT OF \\ INDIVIDUALS WITH FACIAL ANOMALIES
}

\section{Andrea D. Stephan ${ }^{1}$}

STEPHAN, A.D. O desenvolvimento psicossocial e educacional de indivíduos com anomalias faciais. Rev. Bras. Cresc. Desenv. Hum., São Paulo, 13(2): 54-58, 2003.

Resumo: Esta revisão reune dados da literatura científica atual sobre o desenvolvimento psicossocial e educacional de indivíduos portadores de anomalias craniofaciais². vÉ possível observar uma tendência, no tratamento desses indivíduos, de focalizar todos os níveis de complexidade, não apenas objeti ando o seu restabelecimento funcional e anatómico, mas também o desenvolvimento de uma pessoa saudável, no conceito mais amplo de bem-estar emocional, social e profissional. Para que esse objetivo seja plenamente alcançado? é imprescindível uma atenção educacional inclusive e propulsora, com o envolvimento de todos os profissionais. sejam da área da saúde ou da área educacional, em convívio com esses pacientes.

Palavras-chave: anomalia; lábio fissurado; promoção da saúde; educação em saúde.

\section{INTRODUCÃO}

Cada vez mais o ser humano é abordado como um todo: na Odontologia, Medicina, Enfermagem e na Saúde em geral, assim como na Educação e em todas as outras áreas do conhecimento. Essas áreas corroboram para um mesmo objetivo: o desenvolvimento de pessoas saudáveis, física, psicológica e socialmente. Assim sendo, a abordagem integralizada requer a união das diversas especialidades na busca da interdisciplinaridade para que esse objetivo seja alcançado em sua plenitude.

Este artigo propõe focalizar os indivíduos com diferenças faciais (conceito que engloba todos os pacientes que apresentem alguma anomalia congênita ou adquirida), com ênfase nos pa- cientes portadores de fissuras labiais ou lábio-palatais, nos aspectos que concemem ao seu desenvolvimento psicossocial e educacional, ressaltando o papel-chave dos educadores no desenvolvimento saudável desses indivíduos e a importância do apoio familiar.

\section{REVISÃO DA LITERATURA/ DISCUSSÃO}

\section{Promoção de Saúde versus Anomalias Faciais}

O conceito de anomalia craniofacial engloba toda alteração (ou defeito) congênito que envolve a região do crânio e da face (HRAC/USPCENTRINHO, 2003), sendo a fissura de lábio e/ ou de palato uma das quatro formas mais comuns

1 Cirurgia-Dentista; Mestranda em Educação pela Pontificia Universidade Católica do Rio Crandc do Sul - PUCRS. Programa de Pós-Graduaçào em Educação; e Secretaria Municipal de Saúde de Porto Alcgre, Unidade Sanitária Farrapos. Endereço para correspondência: Av. Brasiliano de Morais, 422, Passo D’Areia, Porto Alcgre - RS, CEP 91030-000, tel: 0(XX51) 3341-6363. 0(XX51) 9806-0099. Endereço eletrônico: astephan@hotnet.net

2 Neste artigo, os termos anomalia craniofacial, condição craniofacial e diferença facial serão usados como Sinônimos. 
de anomalias do recém-nascido, sendo outras a Síndromc de Down, defeitos nas mãos e problemas cardiovasculares (MOTOYAMA e col., 2000). Dados epidemiológicos apontam uma incidência da fissura de 1:650 recém-nascidos no Brasil (HRAC/USP-CENTRINHO, 2003; OLIVEIRA e col., 1996); considerando-se que 15.000 crianças nascem por hora no mundo, registra-se o nascimento de um bebê fissurado a cada 2 minutos e meio (WHO, 2002).

A etiologia dessa entidade patológica vem sendo estudada exaustivamente, principalinente no campo da genética, diferenciando-se as expressões sindrômicas das não-sindrômicas, sendo considerada, até o presente momento, como inultifatorial, com fatores genéticos e ambientais atuando em conjunto ou isoladamente (MOTOYAMA e col., 2000).

Inúmeras pesquisas têm sido direcionadas aos indivíduos portadores de diferença craniofacial nos aspectos que concernem às suas particularidades anatômicas e funcionais e como essas influenciam a vida dos indivíduos alistados. Na revisão da literatura, é possível constatar que a presença de diferenças faciais sempre marcam e influenciam de maneira positiva ou negativa a vida dos pacientes e de suas famílias (BERK e col., 2001; BRODER e col., 1998; CHAPPLE \& NUNN, 2001; EISERMAN, 2001; ELIASON e col., 1991; ENDRIGA; KAPP-SIMON, 1999; KELTON, 2001; KENEALY e col., 1988; MARCUSSON e col., 2001; MILLARD \& RICHMAN, 2001; MOURADIAN, 2001; OMOTE, 1993/1994; PAUL \& BRANDT, 1998; SPIRI \& LEITE, 1999; STRAUSS, 2001).

Qual, efetivamente, é a natureza dessas influências? como podem ser classificadas? são permanentes? - eis algumas das muitas perguntas ainda sem resposta que merecem mais atenção.

As dificuldades enfrentadas por pessoas afetadas por anomalias faciais são muitas e vêm sendo estudadas ao longo do tempo. Dependendo da complexidade de sua condição craniofacial, as crianças portadoras são submetidas, em muitos casos, a múltiplos tratamentos e procedimentos cirúrgicos, que as acoinpanham durante toda a infância, a juventude e, algumas vezes, a vida adulta. O tratamento envolve uma equipe multidisciplinar da qual participam: o profissional ortodontista, cirurgião bucomaxilofacial, protesista, odontopediatra, cirurgião plástico, cardiologista, neonatologista, pediatra, psiquiatra, foniatra, otorrinolaringologista, geneticista, fonoaudiólogo, psicólogo, assistente social (MOTOYAMA e col., 2000). Além disso, o/a enfermeiro(a) e, se necessário, ainda outros profissionais, podem participar dessa equipe. A car- ga referente ao tratamento, considerando-se cirurgias, consultas e demais atividades, pode-se tornar imensa, implicando dor, sofrimento, interrupções na rotina da vida familiar, escola e emprego, o que poderá surtir também um efeito adverso no sentido de afetar o senso de autodeterminação e sentido de controle, pelo papel de dependência em que o paciente é colocado (WHO, 2002). O modo como pacientes (crianças), enfrentando todos estes desafios inerentes à sua condição craniofacial, tornam-se adultos saudáveis, não apenas com relação a uma função e anatomia faciais satisfatórios, mas num conceito mais amplo de bem-estar emocional, social e profissional, tem sido o alvo de novas áreas de pesquisa. Observa-se, portanto, uma tendência na ciência da saúde craniofacial - que se caracteriza como promoção de saúde -, que recomenda a análise qualitativa de casos (EISERMAN, 2001; MOURADIAN, 2001), como um instrumento adicional, ao trazer mais entendimento para um campo até agora insuficientemente explorado. Resultam relatos de experiências que podem ajudar futuras gerações de pacientes com anomalias faciais, exemplificando como vencer adversidades, mesmo na presença de severos impedimentos/desvantagens. E importante ressaltar que cada caso é marcado pela história do paciente que pode ter resultado de padrões específicos: a interação do indivíduo com o meio no qual ele está inserido; as influências familiares e educacionais, a realidade socioeconômica e o acesso a diversas formas de tratamento de sua condição craniofacial. No entanto, considerar as diferenças faciais num continuum de saúde e doença, e não apenas como sendo uma deficiência (EISERMAN, 2001), pode ser considerado um grande avanço.

\section{Desenvolvimento Psicossocial e Educacional}

O desenvolvimento deve ser considerado um fenômeno contínuo e ininterrupto, incluíndo todos os tipos de mudanças experimentadas pelos seres humanos. A vida humana apresenta momentos de crescimento extraordinário e também de declínio (MOSQUERA \& STOBAUS,1984) sendo parte de um processo. Indivíduos com diferenças faciais vivem o mesmo processo. No entanto, eles, muitas vezes, atravessam a infância e a juventude enfrentando prolongados tratamentos de suas diferenças anatômicas, funcionais e estéticas. Associadas a tais diferenças, surgem diversos desafios: altos e baixos psicológicos e educacionais a serem superados. Especialmente o desenvolvimento educacional de pacientes com diferenças faciais pode, algumas vezes, ser influenciado pelo pré-julgamento de terceiros. Vários estudos mostram que a atratividade facial pode 
influenciar a avaliação de indivíduos afetados (ELIASON e col., 1991; KENEALY e col., 1988; OMOTE, 1993/1994; SPIRI \& LEITE, 1999), com relação a itens como sociabilidade, popularidade, inteligência acadêmica, autoconfiança e características de liderança (KENEALY e col., 1988). Desse modo, indivíduos afetados podem necessitar de mais empenho para superar expectativas negativas e estigmatizações por parte de terceiros. Somam-se a isso distúrbios de auto-estima, principalmente, na sociedade moderna que valoriza o corpo perfèito como saudável e atraente, em que os portadores de fissuras enfrentam o estigma de carregar um defeito físico como parte de sua identidade (ANDRADE \& ANGERAM 1, 2001). Um estudo dinamarquês relata uma taxa de suicídio entre pacientes fissurados adultos com idade média de 28 anos, duas vezes a da população em geral, sendo de risco especialmente os pacientes que tinham sinais visíveis. (HERSKIND e col., 1993 apud THOMAS e col., 1997, p. 230). No entanto, tem sido afirmado que individuos afetados não desenvolvem uma personalidade psicopatológica (BRODER, 2001; ENDRIGA \& KAPP-SIMON, 1999; WIETHAEUPER, 1994). Uma estrutura familiar positiva e de apoio pode ser um fator determinante neste sentido.

Outros estudos dos anos 70 demonstraram que adultos com fissuras tratadas têm menos probabilidade de se casarem (MCWILLIAMS \& PARADISE, 1973 e PETER \& CHINSKY, 1974, apud THOMAS e col., 1997, p. 230). Porém, torna-se necessária uma reflexão sobre tais dados à luz da atualidade, pois é importante considerar que as formas de tratamento e a abordagem interdisciplinar do paciente têm evoluido positivamente, assim como o conhecimento sobre esta anomalia e suas repercussões na saúde do paciente como um todo têm aumentado consideravelmente, de modo que esses dados podem já não mais corresponder ao momento atual.

BRODER e col. (1998) concluem que crianças fissuradas apresentam risco de baixo rendimento escolar. Resultados dessa natureza devem ser analisados cuidadosamente, pois há muitas variáveis presentes como, por exemplo, o sistema educacional e seu acolhimento, e não se pode considerar que seja uma característica inerente a todos os indivíduos facialmente diferentes. Os mesmos autores sugerem que estratégias psicoeducacionais efetivas sejam desenvolvidas para facilitar o processo de aprendizagem, respeitando e endereçando as particularidades de tais crianças.

Outro aspecto, estudado por BERK e col. (2001), é a ansiedade social de adultos com fissuras orofaciais. Os autores mencionam que essa ansiedade pode funcionar como um mecanismo de proteção estabelecido pelos indivíduos frente a situações vistas como potencialmente amedrontadoras no convívio social, apontando que o desenvolvimento psicossocial de adultos afetados requer mais investigação.

ENDRIGA e KAPP-SIMON (1999) reafirmam o papel dos pais/responsáveis no que se refere ao desenvolvimento de bases psicológicas saudáveis para a criança. Segundo esses mesmos autores, as informações e os dados sobre as habilidades intelectuais e neuropsicológicas de adultos com diferenças craniofaciais são raras.

Com relacão à qualidade de vida de adultos com fissura completa de palato e lábio tratada, MARCUSSON e col. (2001) concluem que um impacto significativo no que diz respeito a aspectos globais, tais como bem-estar e vida social, foi percebido pelos pacientes.

Surge então a dúvida: necessariamente persistem seqüelas comportamentais e cognitivas na vida adulta de indivíduos que têm ou tiveram alguma condição craniofacial?

ENDRIGA e KAPP-SIMON (1999) recomendam que o objetivo do tratamento de crianças com diferenças craniofaciais seja o desenvolvimento de um adulto funcional e psicologicamente saudável, com intervenções médicas regulares e atendimento psicológico quando necessário, completado pela formação educacional em escolas regulares. Individuos facialmente diferentes devem ser incluidos em turmas regulares e esforços devem ser feitos para que um desenvolvimento acadêmico, socioemocional e pessoal seja alcançado, objetivando maximizar o potencial não apenas desta criança-aluno, mas da criança-todo (STOBAUS \& MOSQUERA, 2000).

MOSQUERA e STOBAUS (2001) chamam a atenção sobre como a Saúde está próxima do fenômeno educativo, já que está intimamente ligada à própria pessoa, ao seu corpo, à maneira como cuida dele e ao estilo de vida que leva.

Dessa maneira, pluralismo e multiculturalismo devem caracterizar as diretrizes educacionais do terceiro milênio. Devem prevalecer a tolerância e a aceitação do outro, como um caminho para elucidar o quanto os indivíduos são diferentes e similares ao mesmo tempo (MOSQUERA, 2000).

\section{CONSIDERAÇÕES FINAIS E PROPOSTAS}

Analisa-se, atualmente, a capacidade de superação dos indivíduos com anomalias faciais dos problemas inerentes à sua condição e de que maneira essa capacidade pode ser potencializada. 
Baseado na apresentação de dados que seguramente não esgotam o assunto, que é de natureza multidisciplinar, chama-se a atenção para as três dimensões nas quais a Educação pode desempenhar um papel fundamental:

1. O desenvolvimento educacional dos indivíduos afetados, sendo a Educação o meio através do qual esses pacientes podem se tornar pessoas autônomas e independentes. Os estudos de linguística cognitiva, até o presente momento, não indicam diferença significativa na capacidade psicolingüística dos portadores de diferença facial.

2. A conscientização de pais e responsáveis com relação à desestigmatização da diferença facial, às formas de tratamento e às implicações conseqüentes das mesmas e ao papel de motivadores que eles podem desempenhar frente aos pacientes. O otimismo das pessoas envolvidas pode mobilizar os indivíduos afetados no sentido de ação pessoal e profissional.

3. Familiarização de professores quanto às condições craniofaciais quanto às suas implicações, para que se sintam preparados para re- ceber, na sociedade educacional, indivíduos afetados, de forma a incluí-los em classes regulares.

A Educaçao, portanto, não deve ficar em segundo plano coin relação ao extenso tratamento ao qual os pacientes são submetidos, pois o tratamento implica objetivos que vão aléin da correção da patologia.

Outrossiin, é preciso a aceitação de que fatores de tensão relacionados à condição craniofacial estejam presentes. Grupos de apoio para pacientes e seus pais/responsáveis, com a participação de portadores de diferença facial que possam relatar suas experiências de como enfrentaram o desafio e mostrar como é possível ter-se um desenvolvimento psicossocial e educacional positivo, podem ser de grande auxílio.

\section{A GRADECIMENTO}

À Professora Sandra Rico, do Instituto de Cultura Hispânica da PUC/RS, pela revisão do texto.

\begin{abstract}
This study aims at researching the existing scientific literature about psychosocial and educational outcomes of individuais with craniofacial anomalies. It is possible to observe a new trend in the treatment of these individuais, focusing on ali ieveis of complexity, aiming $\mathrm{t} \wedge \mathrm{l} \wedge \mathrm{Ot}$ oniy at functionai and anatomical restoring, but aiso at the development of a healthy person in the broader concept of emotional, social and professionai weii-being. To reach this goal. special educational attention, inclusive and propulsive. is necessary, with the involvement of ali professionals in contact with these patients. from health care and educational arcas.
\end{abstract}

Key-worels: abnormality; cleft lip; health promotion; health education.

\section{REFERÊNCIAS BIBLIOGRÁFICAS}

ANDRADE, D.; DE: ANGERAMI, E. L. S. A auto-estima em adolescentes com e sem fissuras de lábio e/ou de palato. Revista Latino-Americana de Enfermagem, 9(6): 37-41, nov.- dez. 2001.

BERK, N. W.; COOPER, M. E.; LIU, Y.; MARAZITA, M. L. Social anxiety in Chinese adults with oral-facial clefts. Cleft PalateCraniofacial Journal, 38(2): 126-133, March 2001.

BRODER, H. L. Using psychological assessment and therapeutic strategies to enhance well-being. Cleft Palate-Craniofacial Journal, 38(3): 248-254, May 2001.

BRODER, H. L.; RICHMAN, L. C.; MATHESON, P. B. Learning disability, school achievemente, and grade retention among children with cleft: a twocenter study. Cleft PalateCraniofacial Journal. 15(2): 127-131,March 1998.
CHARRLE, J. R.; NUNN, J. H. The oral health of children with clefts of the lip, palate, or both. Cleft Palate-Craniofacial Journal. 38(5): 525-528, September 2001.

EISERMAN, W. Unique outcomes and positive contributions associated with facial diference: Expanding research and practice. Cleft Palate-Craniofacial Journal, 38(3): 230-244, May 2001.

ELIASON, M. I.; HARDIN, M. A.; OLIN, W. H. I actors that influence ratings of facial appearance for children with cleft lip and palate. Cleft Palate-Craniofacial Journal, 28(2): 190-194, April 1991.

ENDRIGA, M. C.; KAPP-SIMON, K. A. Psychological issues in craniofacial care: state of the art. Cleft Palate-Craniofacial Journal, 36(1): 1-11, January 1999. 
HRAC/USP-CENTRINHO. Ciência e humanizacão de mãos dadas. Bauru, 2003. Disponível em: <http://www.centrinho.usp.br/>. Acesso em: 18 abr. 2003.

KELTON, R.W. Racing up to stigma: Workplace and personal strategies. Cleft PalateCraniofacial Journal, 38(3): 245-247, May 2001.

KENEALY, P.; FRUDE, N.; SHAW, W. Influence of children's physical attractiveness on teachcr expectations. The Journal of Social Psychology. 128(3): 373-383, June 1988.

MARCUSSON, A.; AKERLIND, I.; PAULIN, G. Quality of life in adults with repaired complete cleft lip and palate. Cleft PalateCraniofacial Journal, 38(4): 379-385, july 2001.

MILLARD, T.; RICHMAN, L. C. Different cleft conditions, facial appearance, and speech: Relationship to psychological variables. Cleft Palate-Craniofacial Journal. 38(1): 68-75, January 2001.

MOSQUERA, J. J. M. Pós-modernidade, cultura e professorado: uma análise da subjetividade docente. Educação, Porto Alegre, 23 ^(41): 31 -46, ago. 2000.

MOSQUERA, J. J. M.; STOBAUS, C. D. Educação para a .saúde. Desafio para socieelades em mudança. $2^{\mathrm{a}}$ ed. Porto Alegre, D.C. Luzatto, 1984.

MOSQUERA, J. J. M.; STOBAUS, C. D. Educação, saúde e drogodependência: uma relação crítica. Educação, Porto Alegre, 24(45): 3348, nov. 2001.

MOTOYAMA, L. C. J.; LINO, A. P.; ZAMBON JR, D.; LOPES., L. D. Tratamento ortodôntico em pacientes com fissuras lábiopalatinas. Revista Paulista ele Odontologia, 22(2): 4-10, mar./abr. 2000.

MOURADIAN, W. E. Deficits versus strengths: Ethics and implications for clinical practice and research. Cleft Palate-Craniofacial Journal. 38(3): 255-259, May 2001.
OLIVEIRA, D. F. B.; CAPELOZZA, A. L. A.; CARVALHO, I. M. M. Alterações de desenvolvimento dentário em fissurados. Revista da APCD, 50(1): 83-86, jan./fev. 1996.

OMOTE, S. Atratividade fisica facial e percepção de degficiências. Dielática, São Paulo, 29: 115-124, 1993/1994.

PAUL, T.; BRANDT, R. S. Oral and dental health status of children with cleft lip and or palate. Cleft Palate-Craniofacial Journal. 35(4): 329-332, Jul. 1998.

SPIRI, W. C.; LEITE, M. M. J. Convivendo com o portador de fissura lábio-palatal: o vivencial da enfermeira. Rev. Esc. Enf USP, 33(1): 81-94, mar. 1999.

STOBAUS, C. D.; MOSQUERA, J. tM. Educação inclusiva: um novo olhar sobre educação especial. Educação, Porto Alegre, 23(42^): 129-142, nov. 2000.

STRAUSS, R.P. “Only skin deep”: Health, resilience, and craniofacial care. Cleft PalateCraniofacial Journal, 38^(3^): 226-229, May 200R

THOMAS, P. T.; TURNER, S. R.: RUMSEY, N.; DOWELL, T.; SANDY, J. R. Satisfaction with facial appcarance among subjects sffected by a cleft. Cleft Palate-Craniofacial Journal. 34(3): 226-231, May 1997.

WHO. Global strategies to reduce the health-care burden of craniofacial anomalies. Geneva, 2002. Disponível em: $<$ http:// www.who.int/ncd/hgn/ cfa8-anx.pdf $>$. Acesso em 18. abr. 2003.

WIETHAEUPER, D. Atratividade física facial e estigmatização em crianças portadoras de fissuras labiais. Porto Alegre, 1994. [Dissertaçào de Mestrado - Instituto de Psicologia, Pontifícia Universidade Católica do Rio Grande do Sul].

Recebido em 26/03/2003

Modifcado em 12/05/2003

Aprovado em 05/09/2003 